EPJ manuscript No.

(will be inserted by the editor)

CERN-PH-TH/2004-061

TTP04-07

\title{
Radiative return at $\Phi$ - and $B$-factories: FSR for muon pair production at next-to-leading order *
}

\author{
Henryk Czyż ${ }^{1}$ a, Agnieszka Grzelińska ${ }^{1}$ b, Johann H. Kühn² c , and Germán Rodrigo ${ }^{3,4 ~ d ~}$ \\ 1 Institute of Physics, University of Silesia, PL-40007 Katowice, Poland. \\ 2 Institut für Theoretische Teilchenphysik, Universität Karlsruhe, D-76128 Karlsruhe, Germany. \\ 3 Department of Physics, Theory Division, CERN, CH-1211 Geneva 23, Switzerland. \\ ' 4 Instituto de Física Corpuscular, E-46071 Valencia, Spain.
}

April 7, 2004

\begin{abstract}
Muon pair production through the radiative return is of importance for a measurement of the hadronic production cross section in two ways: it provides an independent calibration and it may give rise to an important background for a measurement of the pion form factor. With this motivation the Monte Carlo event generator PHOKHARA is extended to include next-to-leading order radiative corrections to the reaction $e^{+} e^{-} \rightarrow \mu^{+} \mu^{-} \gamma$. Furthermore, virtual ISR corrections to FSR from pions are introduced, which extends the applicability of the generator into a new kinematical regime. Finally, the effect of photon vacuum polarization is introduced into this new version of the generator.
\end{abstract}

\section{Introduction}

The high luminosity of currently operating $\Phi$ - and $B$ meson factories offers the unique opportunity to measure the famous ratio $R \equiv \sigma\left(e^{+} e^{-} \rightarrow\right.$ hadrons $) / \sigma_{\text {point }}$ over a wide range of energies in one single experiment, through the radiative return. This possibility was suggested a long time ago for pion-pair production [1]. In view of the capabilities of the currently operating machines, the idea was revived in 2, where a Monte Carlo event generator EVA was constructed, which simulates the production of a pion pair plus one hard photon from initial- and final-state radiation (ISR and FSR). The program includes additional collinear radiation from the incoming electrons through structure function techniques and was used to demonstrate the feasibility of such a measurement in ongoing experiments.

* Work supported in part by BMBF under grant number 05HT9VKB0, EC 5th Framework Programme under contract HPRN-CT-2002-00311 (EURIDICE network), TARI project HPRI-CT-1999-00088, Polish State Committee for Scientific Research (KBN) under contract 2 P03B 017 24, BFM200200568, Generalitat Valenciana under grant GRUPOS03/013, and MCyT under grant FPA-2001-3031.

a e-mail: czyz@us.edu.pl

b e-mail: agrzel@us.edu.pl

c e-mail: johann.kuehn@uni-karlsruhe.de

d e-mail: german.rodrigo@cern.ch
The generator was subsequently extended to simulate the production of other hadronic final states: four pions in [3 4] and nucleon pairs in [5], as well as muons [6]. The influence of collinear lepton pair radiation on these measurements was investigated in [7.

An important step forward was the evaluation of the complete one-loop corrections to ISR 8,9 and the construction of the event generator PHOKHARA [6], which included these virtual corrections and the corresponding radiation of two photons. PHOKHARA, like EVA, can be easily used to simulate reactions with arbitrary hadronic final states, once a specific model has been chosen for the relevant matrix element of the hadronic current; it is currently available for $2 \pi, 4 \pi, p \bar{p}$ and $n \bar{n}$ production 6 6, 4,5] and of course for muon pairs. A number of cross section measurements based on the radiative return, which have made use of simulations based on EVA and PHOKHARA, have been presented recently: a precise measurement of the pion form factor made by the KLOE Collaboration [10. 11,12 13; preliminary results on four-prong final states have been obtained by the BABAR Collaboration 14. Extensive discussions of theoretical and experimental aspects of the radiative return, including various topics not covered by the present paper, can be found in the literature 15, 16, 17, 18, 19 20, 21, 22 23 24, 25, 26, 27, 28, 29, 30]; considerations related to the radiative return can be found in 31,32; perspectives of its use outside hadronic cross section measurements and the long term potential of the method are outlined in 33 . 
Considering measurements with a precision close to one percent, FSR starts to become an issue, in particular the contribution from the two-step process $e^{+} e^{-} \rightarrow$ $\gamma \gamma^{*}\left(\rightarrow \pi^{+} \pi^{-} \gamma\right)$ [18. FSR has to be modeled separately for every different mode. In leading order ( $\mathrm{LO})$, it is now included in PHOKHARA for the $\pi^{+} \pi^{-}$and the $\mu^{+} \mu^{-}$ modes, in next-to-leading order (NLO), i.e. for the twostep process with one hard photon from ISR and real plus virtual radiation from the final state for $\pi^{+} \pi^{-}$only.

It is the purpose of the present paper to extend the same approach to final states with muon pairs. This is motivated, on the one hand, by the fact that this reaction is an important background for the pion form factor measurement, on the other hand, that it may be used for a measurement of the ratio between hadronic final states and muon pairs of the same invariant mass. This might lead to a direct determination of the $R$-ratio, with the cancellation of many uncertainties that arise in the absolute cross section determination.

Although, technically speaking, the treatment of muons is quite similar to that of pions, a number of physics aspects are quite different. First of all, in contrast to radiation from muons, the amplitude for photon radiation from pions can only be parametrized by a model form factor, and the model dependence becomes particularly relevant for energetic photons. Then, at larger cms energies, relevant e.g. to the $B$-factories, the leading order FSR with only one photon in the final state is strongly suppressed in the pion case, as a consequence of the pion form factor, whereas the point-like behaviour of the muon leads to important contributions from ISR as well as FSR in leading order.

In order to arrive at a full result for muon pair production at NLO, corrections with combined virtual emission from the initial state and real hard emission from the final state (Fig. 1c) must be included, and combined with those from "simultaneous" soft real ISR and hard FSR (Fig. 1a). To offer a uniform program, the same contributions will also be included for pion pair production. However, we will demonstrate that their effect is small already for KLOE energies, in particular for the standard KLOE cuts, and completely irrelevant for higher energies.

Finally we study the influence of the leptonic and hadronic vacuum polarization on the radiative return, where we adopt the parametrization suggested in 34. For the measurement of the integrated cross section with cuts suppressing FSR, vacuum polarization just leads to a multiplicative factor that can be taken into account by correcting the result at the end of the experimental analysis. The forward-backward or the charge asymmetry, however, is affected by vacuum polarization effects in a non-trivial manner.

Let us briefly outline the content of this paper. The amplitudes for real and virtual radiation in NLO for muon pair production, which are the main additional ingredients in the new program, will be introduced in Section 2. Tests of the technical precision of the program and some of its physics results will be presented in Section 3. The ISR correction to real hard FSR for the $\pi^{+} \pi^{-}$mode will be intro-

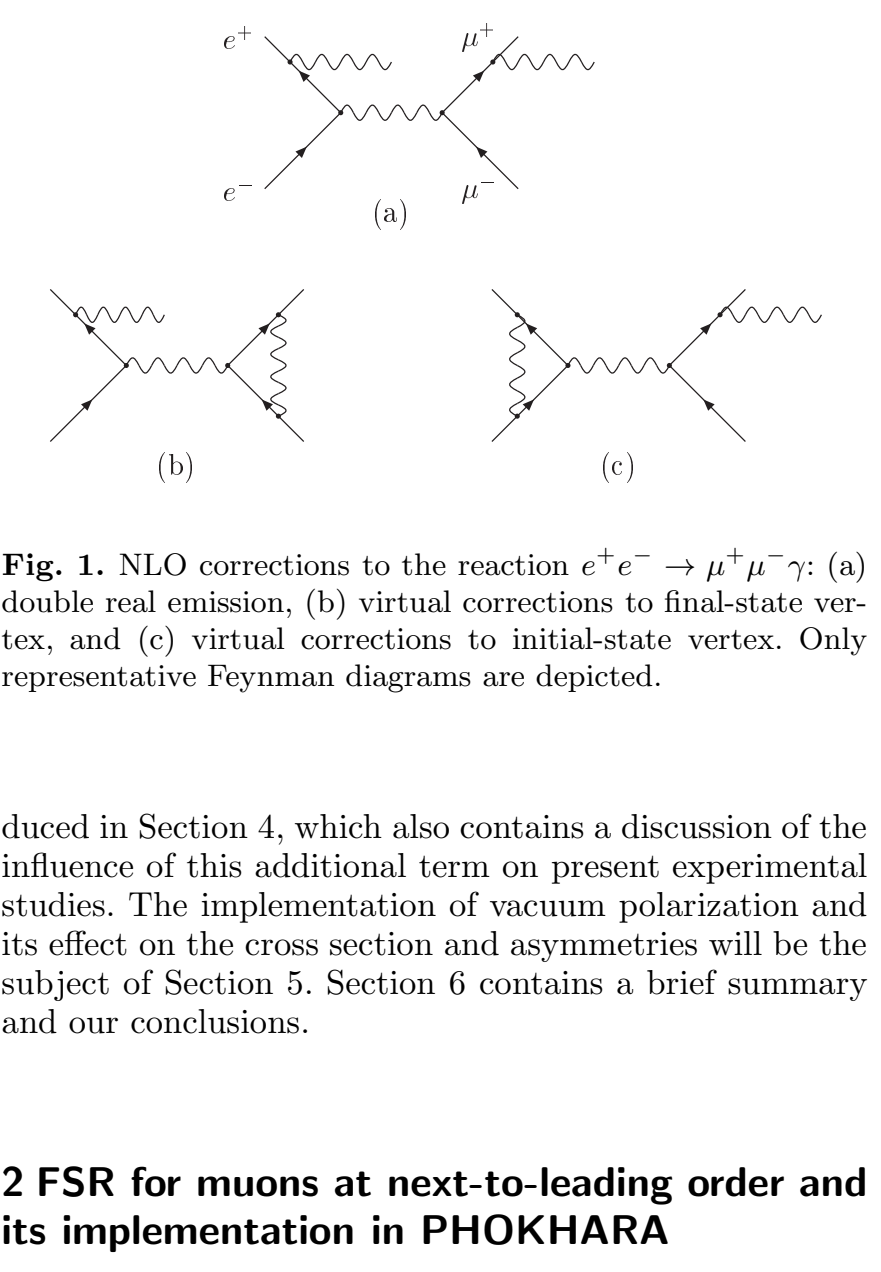

The most relevant contributions to FSR at NLO, which are depicted schematically in Fig. 11 consist of double real photon emission diagrams, where one photon is emitted off the initial-state leptons and the other is emitted from the final state (Fig. 19), final-state vertex corrections to single initial-state photon emission (Fig. 10), and initialstate vertex corrections to single final-state photon emission (Fig. 11).

The amplitude for the process depicted in Fig. 11

$$
\begin{aligned}
& e^{+}\left(p_{1}, \lambda_{e^{+}}\right)+e^{-}\left(p_{2}, \lambda_{e^{-}}\right) \rightarrow \\
& \mu^{+}\left(q_{1}, \lambda_{\mu^{+}}\right)+\mu^{-}\left(q_{2}, \lambda_{\mu^{-}}\right)+\gamma\left(k_{1}, \lambda_{1}\right)+\gamma\left(k_{2}, \lambda_{2}\right)
\end{aligned}
$$

is given by

$$
\begin{aligned}
& M_{\mathrm{IFSNLO}}^{\mathrm{H}}\left(\lambda_{e^{+}}, \lambda_{e^{-}}, \lambda_{\mu^{+}}, \lambda_{\mu^{-}}, \lambda_{1}, \lambda_{2}\right)=-\frac{(4 \pi \alpha)^{3 / 2}}{\hat{Q}^{2}}\{ \\
& v_{I}^{\dagger}\left(p_{1}, \lambda_{e^{+}}\right) A\left(\lambda_{\mu^{+}}, \lambda_{\mu^{-}}, \lambda_{1}, \lambda_{2}\right) u_{I}\left(p_{2}, \lambda_{e^{-}}\right) \\
& \left.+v_{I I}^{\dagger}\left(p_{1}, \lambda_{e^{+}}\right) B\left(\lambda_{\mu^{+}}, \lambda_{\mu^{-}}, \lambda_{1}, \lambda_{2}\right) u_{I I}\left(p_{2}, \lambda_{e^{-}}\right)\right\} \\
& +\quad\left(k_{1} \leftrightarrow k_{2}, \lambda_{1} \leftrightarrow \lambda_{2}\right)
\end{aligned}
$$


where the index $\mathrm{H}$ indicates that both photons are hard,

$$
\begin{aligned}
& A\left(\lambda_{\mu^{+}}, \lambda_{\mu^{-}}, \lambda_{1}, \lambda_{2}\right)= \\
& \frac{\left(\varepsilon^{*}\left(k_{1}, \lambda_{1}\right)^{-} k_{1}^{+}-2 \varepsilon^{*}\left(k_{1}, \lambda_{1}\right) \cdot p_{1}\right) D^{-}\left(\lambda_{2}, \lambda_{\mu^{+}}, \lambda_{\mu^{-}}\right)}{2 k_{1} \cdot p_{1}} \\
& +\frac{D^{-}\left(\lambda_{2}, \lambda_{\mu^{+}}, \lambda_{\mu^{-}}\right)\left(2 \varepsilon^{*}\left(k_{1}, \lambda_{1}\right) \cdot p_{2}-k_{1}^{+} \varepsilon^{*}\left(k_{1}, \lambda_{1}\right)^{-}\right)}{2 k_{1} \cdot p_{2}},
\end{aligned}
$$

and

$$
\begin{aligned}
& B\left(\lambda_{\mu^{+}}, \lambda_{\mu^{-}}, \lambda_{1}, \lambda_{2}\right)= \\
& \frac{\left(\varepsilon^{*}\left(k_{1}, \lambda_{1}\right)^{+} k_{1}^{-}-2 \varepsilon^{*}\left(k_{1}, \lambda_{1}\right) \cdot p_{1}\right) D^{+}\left(\lambda_{2}, \lambda_{\mu^{+}}, \lambda_{\mu^{-}}\right)}{2 k_{1} \cdot p_{1}} \\
& +\frac{D^{+}\left(\lambda_{2}, \lambda_{\mu^{+}}, \lambda_{\mu^{-}}\right)\left(2 \varepsilon^{*}\left(k_{1}, \lambda_{1}\right) \cdot p_{2}-k_{1}^{-} \varepsilon^{*}\left(k_{1}, \lambda_{1}\right)^{+}\right)}{2 k_{1} \cdot p_{2}},
\end{aligned}
$$

with

$$
\hat{Q}=p_{1}+p_{2}-k_{1}=q_{1}+q_{2}+k_{2}, \quad s^{\prime}=\hat{Q}^{2} .
$$

$D^{\mu}$ is the current describing the $\mu^{+} \mu^{-} \gamma$ final state [4, which we report below for completeness

$$
\begin{aligned}
D^{\mu}\left(\lambda_{2}, \lambda_{\mu^{+}}, \lambda_{\mu^{-}}\right) & =i e\left\{u_{I}^{\dagger}\left(q_{2}, \lambda_{\mu^{-}}\right) \tilde{A}^{\mu}\left(\lambda_{2}\right) v_{I}\left(q_{1}, \lambda_{\mu^{+}}\right)\right. \\
& \left.+u_{I I}^{\dagger}\left(q_{2}, \lambda_{\mu^{-}}\right) \tilde{B}^{\mu}\left(\lambda_{2}\right) v_{I I}\left(q_{1}, \lambda_{\mu^{+}}\right)\right\},
\end{aligned}
$$

with

$$
\begin{aligned}
\tilde{A}^{\mu}\left(\lambda_{2}\right) & =\frac{\left(2 q_{2} \cdot \varepsilon^{*}\left(k_{2}, \lambda_{2}\right)+\varepsilon^{*}\left(k_{2}, \lambda_{2}\right)^{-} k_{2}^{+}\right) \sigma^{\mu-}}{2 k_{2} \cdot q_{2}} \\
& -\frac{\sigma^{\mu-}\left(2 q_{1} \cdot \varepsilon^{*}\left(k_{2}, \lambda_{2}\right)+k_{2}^{+} \varepsilon^{*}\left(k_{2}, \lambda_{2}\right)^{-}\right)}{2 k_{2} \cdot q_{1}}, \\
\tilde{B}^{\mu}\left(\lambda_{2}\right) & =\frac{\left(2 q_{2} \cdot \varepsilon^{*}\left(k_{2}, \lambda_{2}\right)+\varepsilon^{*}\left(k_{2}, \lambda_{2}\right)^{+} k_{2}^{-}\right) \sigma^{\mu+}}{2 k_{2} \cdot q_{2}} \\
& -\frac{\sigma^{\mu+}\left(2 q_{1} \cdot \varepsilon^{*}\left(k_{2}, \lambda_{2}\right)+k_{2}^{-} \varepsilon^{*}\left(k_{2}, \lambda_{2}\right)^{+}\right)}{2 k_{2} \cdot q_{1}},
\end{aligned}
$$

and $\sigma^{\mu \pm}=\left(I, \pm \sigma_{i}\right)$, where $\sigma_{i}, i=1,2,3$, are the Pauli matrices and $a^{ \pm}=a^{\mu} \sigma_{\mu}^{ \pm}$for any four-vector $a^{\mu}$.

The virtual (Fig. 1b) plus soft photon corrections to the final-state vertex can be written as 35.

$$
d \sigma_{\mathrm{IFSNLO}, \mathrm{f}}^{\mathrm{V}+\mathrm{S}}=\frac{\alpha}{\pi} \eta^{\mathrm{V}+\mathrm{S}}\left(s^{\prime}, E_{2}^{\mathrm{cut}}\right) d \sigma_{\mathrm{ISR}}^{(0)}\left(s^{\prime}\right)
$$

where $d \sigma_{\text {ISR }}^{(0)}$ is the leading order $e^{+} e^{-} \rightarrow \mu^{+} \mu^{-} \gamma$ cross section, with the photon emitted off the initial leptons only, and

$$
\begin{aligned}
& \eta^{\mathrm{V}+\mathrm{S}}\left(s^{\prime}, E_{2}^{\mathrm{cut}}\right)=-2\left[\frac{1+\beta_{\mu}^{2}}{2 \beta_{\mu}} \log (t)+1\right] \log \left(2 w^{\prime}\right) \\
& -\frac{\log (t)}{s^{\prime} \beta_{\mu}}\left[\frac{5}{2} s^{\prime}-7 m_{\mu}^{2}+3 m_{\mu}^{2} x_{\mu}\right]-2+\log \left(\frac{1-\beta_{\mu}^{2}}{4}\right) \\
& -\frac{1+\beta_{\mu}^{2}}{\beta_{\mu}}\left[2 \operatorname{Li}_{2}\left(\frac{2 \beta_{\mu}}{1+\beta_{\mu}}\right)-\frac{\pi^{2}}{2}-\log (t) \log \left(\frac{1+\beta_{\mu}}{2}\right)\right] .
\end{aligned}
$$

$E_{2}^{\text {cut }}$ is the maximal energy of the soft photon in the $s^{\prime}$ rest frame, and

$$
\begin{aligned}
& \beta_{\mu}=\sqrt{1-4 m_{\mu}^{2} / s^{\prime}}, \quad t=\frac{1-\beta_{\mu}}{1+\beta_{\mu}}, \\
& w^{\prime}=E_{2}^{\text {cut }} / \sqrt{s^{\prime}}, \quad \frac{1}{x_{\mu}}=\frac{s^{\prime}}{2 m_{\mu}^{2}}+1 .
\end{aligned}
$$

The virtual (Fig. 1 ) plus soft photon corrections to the initial-state vertex can be written as 36 .

$$
d \sigma_{\mathrm{IFSNLO}, \mathrm{i}}^{\mathrm{V}+\mathrm{S}}=\frac{\alpha}{\pi} \delta^{\mathrm{V}+\mathrm{S}}\left(s, E_{\gamma}^{\mathrm{min}}\right) d \sigma_{\mathrm{FSR}}^{(0)}(s)
$$

where $d \sigma_{\mathrm{FSR}}^{(0)}$ is the leading order $e^{+} e^{-} \rightarrow \mu^{+} \mu^{-} \gamma$ cross section, with the photon emitted off the final leptons only, and, for $m_{e}^{2} \ll s$ :

$$
\delta^{\mathrm{V}+\mathrm{S}}=2\left\{(L-1) \log (2 w)+\frac{3}{4} L-1+\zeta(2)\right\} .
$$

$E_{\gamma}^{\min }$ is the maximal energy of the soft photon (or the minimal energy of the hard photon) in the $s$ rest frame, while

$$
L=\log \left(\frac{s}{m_{e}^{2}}\right) \text { and } w=E_{\gamma}^{\min } / \sqrt{s} .
$$

To match hard, soft and virtual radiation smoothly, the energy cutoff $\left(E_{2}^{\text {cut }}\right)$ has to be transformed from the rest frame of the $\mu^{+} \mu^{-} \gamma$ system (with the photon emitted from the final state) to the laboratory frame $\left(e^{+} e^{-}\right.$cms frame) $\left(E_{\gamma}^{\min }\right)$. In fact it is necessary to recalculate the soft photon contribution, as the cut on $Q^{2}=\left(q_{1}+q_{2}\right)^{2}$ depends in the latter case on the angle between the two emitted photons. Now

$$
\begin{aligned}
& \eta^{\mathrm{V}+\mathrm{S}}\left(s^{\prime}, E_{2}^{\text {cut }}\right)=-2\left[\frac{1+\beta_{\mu}^{2}}{2 \beta_{\mu}} \log (t)+1\right] \\
& \times\left[\log (2 w)+1+\frac{s^{\prime}}{s^{\prime}-s} \log \left(\frac{s}{s^{\prime}}\right)\right]-2+\log \left(\frac{1-\beta_{\mu}^{2}}{4}\right) \\
& -\frac{\log (t)}{s^{\prime} \beta_{\mu}}\left[\frac{5}{2} s^{\prime}-7 m_{\mu}^{2}+3 m_{\mu}^{2} x_{\mu}\right]-\frac{1+\beta_{\mu}^{2}}{\beta_{\mu}}\left[2 \operatorname{Li}_{2}\left(\frac{2 \beta_{\mu}}{1+\beta_{\mu}}\right)\right. \\
& \left.-\frac{\pi^{2}}{2}-\log (t) \log \left(\frac{1+\beta_{\mu}}{2}\right)\right] .
\end{aligned}
$$

Using Eqs. (2) to (15) the implementation of FSR in combination with ISR is straightforward. 
Table 1. Total cross section (nb), corresponding to the $Q^{2}$ distributions from Fig. 2 for the process $e^{+} e^{-} \rightarrow \mu^{+} \mu^{-} \gamma$ at $\mathrm{NLO}$, for different values of the soft photon cutoff.

\begin{tabular}{ccl}
$w$ & $\sqrt{s}=1.02 \mathrm{GeV}$ & $10.52 \mathrm{GeV}$ \\
\hline $10^{-4}$ & $28.237(1)$ & $0.19854(7)$ \\
$10^{-5}$ & $28.236(1)$ & $0.19883(14)$ \\
\hline
\end{tabular}

We have not included diagrams where two photons are emitted from the final state, neither final-state vertex corrections with associated real radiation from the final state [37. This constitutes a radiative correction to FSR and will give non negligible contributions only for those cases, where at least one photon is collinear with one of the muons. Box diagrams with associated real radiation from the initial- or the final-state leptons, as well as pentagon diagrams, are also neglected. As long as one considers charge symmetric observables only, their contribution is neither divergent in the soft nor the collinear limit and thus of order $\alpha / \pi$ without any enhancement factor. We want to stress that we have included only C-even gauge invariant sets of diagrams (see Fig. 9 of Ref. 18 for a graphical representation of the equivalent set of diagrams in pion pair production). The box and pentagon diagrams that we have neglected are related to ISR-FSR interferences, and therefore are suppressed after suitable kinematical cuts used within the radiative return method. One could also neglect some small contributions from the diagrams taken into account, but they are kept for the sake of completeness, as the full 1-loop radiative corrections will be consider in a future publication. At this point these small contributions will become relevant allowing accurate calculations not restricted to radiative return physical configurations.

\section{Tests of the Monte Carlo program and discussion of physical results}

A number of tests were performed to ensure the technical precision of the new version of PHOKHARA. The square of the matrix element, summed over polarizations of the final particles and averaged over polarizations of the initial particles, was calculated with FORM 38, using the standard trace method. External gauge invariance was checked analytically when using the trace method and numerically for the amplitude calculated with the helicity amplitude method. The two results for the square of the matrix element summed over polarizations were compared numerically. The code based on the result from the trace method was written in quadruple precision to reduce cancellations. The code based on the result obtained with the helicity amplitude method uses double precision for real and complex numbers and is now incorporated in the code of PHOKHARA 4.0. Agreement of 13 significant digits (or better) was found between the two codes. The sensitivity of the integrated cross section to the choice of the cutoff $w$ can be deduced from Fig. 2 and Table 1 For simplicity
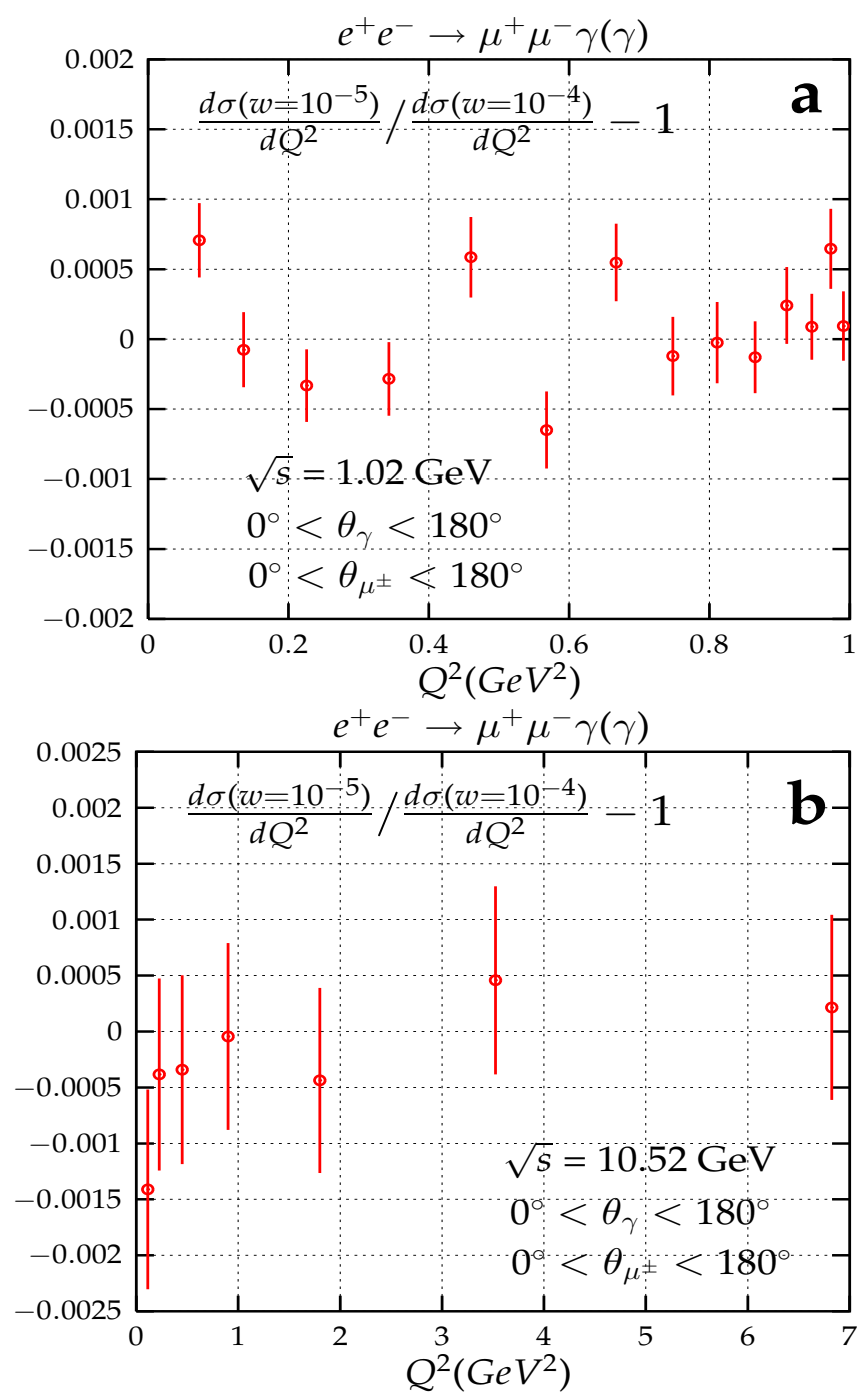

Fig. 2. Comparison of the $Q^{2}$ distribution for two values of the soft photon cutoff $\left(w=10^{-4}\right.$ vs. $\left.10^{-5}\right)$ for $\sqrt{s}=1.02 \mathrm{GeV}$ (a) and $\sqrt{s}=10.52 \mathrm{GeV}$ (b). One of the photons was required to have energy $>10 \mathrm{MeV}$ (for $\sqrt{s}=1.02 \mathrm{GeV}$ ) and $>100 \mathrm{MeV}$ (for $\sqrt{s}=10.52 \mathrm{GeV}$ ). One sigma statistical errors are shown for the ratio of the two distributions.

the same separation parameter $w$ was chosen for ISR and FSR corrections. Choosing $w=10^{-4}$ or less, the result becomes independent of $w$, up to expected systematic differences of order $10^{-4}$. These systematic differences are visible already in Fig. 2a, where $\chi^{2} /$ d.o.f $=30 / 14$, while in Fig. 2 b statistical errors are about $0.1 \%$, and $\chi^{2} /$ d.o.f $=3.5 / 7$. The tests prove that the analytical formula describing soft photon emission as well as the Monte Carlo integration in the soft photon region are well implemented in the program.

For the purpose of further tests we have also calculated the integrated contribution of hard photon emission from the final state to the ISR spectrum. Integrating over all angles and energies, from $E_{2}^{\text {cut }}$ (defined in the $s^{\prime}$ rest frame) to the kinematical limit of the final-state photon, 
we find that this contribution can be written as

$$
d \sigma_{\mathrm{IFSNLO}, \mathrm{f}}^{\mathrm{H}}=\frac{\alpha}{\pi} \eta^{\mathrm{H}}\left(s^{\prime}, E_{2}^{\mathrm{cut}}\right) d \sigma_{\mathrm{ISR}}^{(0)}\left(s^{\prime}\right),
$$

where

$$
\begin{aligned}
& \eta^{\mathrm{H}}\left(s^{\prime}, E_{2}^{\text {cut }}\right)=-\frac{1+\beta_{\mu}^{2}}{\beta_{\mu}}\left[\operatorname{Li}_{2}\left(1-\frac{t_{m}}{t}\right)-\operatorname{Li}_{2}\left(t_{m} t\right)\right. \\
& \left.+\zeta(2)+\frac{\log ^{2}(t)}{2}\right]-\log \left(t_{m}-t\right)\left[\frac{1+\beta_{\mu}^{2}}{\beta_{\mu}} \log \left(\frac{t_{m}}{t}\right)\right. \\
& -2]+\frac{\log \left(t_{m}\right)}{\beta_{\mu}}\left\{\frac{1-\beta_{\mu}^{2}}{1-\beta_{m}^{2}}\left[1+\frac{x_{\mu}\left(2-\beta_{m}^{2}\right)}{\left(1-\beta_{m}^{2}\right)}\right]\right. \\
& +\left(1+\beta_{m}^{2}\right)\left[\log \left(\frac{4 t\left(\beta_{\mu}^{2}-\beta_{m}^{2}\right)}{\left(1-\beta_{\mu}^{2}\right)\left(1-\beta_{m}^{2}\right)}\right)+\frac{\log \left(t_{m}\right)}{2}\right] \\
& \left.-\frac{1}{8}\left[17-\beta_{\mu}^{2}-2 x_{\mu}\right]\right\}-\frac{x_{\mu}}{4 \beta_{m} \beta_{\mu}}\left\{33-17 \beta_{\mu}^{2}\right. \\
& \left.+\frac{1}{1-\beta_{m}^{2}}\left[-27+11 \beta_{\mu}^{2}-\frac{6\left(1-\beta_{\mu}^{2}\right)}{1-\beta_{m}^{2}}\right]\right\} \\
& -2 \log \left(1-t_{m} t\right),
\end{aligned}
$$

$t, x_{\mu}$ and $\beta_{\mu}$ are defined in Eq. (11), and

$$
\beta_{m} \equiv \sqrt{1-\frac{4 m_{\mu}^{2}}{Q_{m}^{2}}}, \quad t_{m}=\frac{1-\beta_{m}}{1+\beta_{m}}
$$

Here $Q_{m}^{2}$ is the maximum value of $Q^{2}$

$$
Q_{m}^{2}=s^{\prime}-2 E_{2}^{\mathrm{cut}} \sqrt{s^{\prime}}
$$

For small $E_{2}^{\text {cut }}, w=E_{2}^{\text {cut }} / \sqrt{s^{\prime}} \ll 1$, the function $\eta^{\mathrm{H}}$ reduces to

$$
\begin{aligned}
& \eta^{\mathrm{H}}\left(s^{\prime}, E_{2}^{\text {cut }}\right) \simeq \log (2 w)\left[2+\frac{1+\beta_{\mu}^{2}}{\beta_{\mu}} \log (t)\right] \\
& +\frac{1+\beta_{\mu}^{2}}{\beta_{\mu}}\left[\operatorname{Li}_{2}\left(t^{2}\right)-\zeta(2)-2 \log (t) \log \left(\frac{1+\beta_{\mu}}{2}\right)\right] \\
& +\frac{\log (t)}{8 \beta_{\mu}}\left[-5+\beta_{\mu}^{2}+6 x_{\mu}\right]-2 \log \left(\frac{4 \beta_{\mu}^{2}}{1-\beta_{\mu}^{2}}\right) \\
& +\frac{3}{2} x_{\mu}+\frac{11}{4} .
\end{aligned}
$$

Adding virtual, soft (Eq. (10) ) and hard (Eq. (18)) corrections, the familiar correction factor 39.

$$
\begin{aligned}
& \eta\left(s^{\prime}\right)=\frac{1+\beta_{\mu}^{2}}{\beta_{\mu}}\left[4 \operatorname{Li}_{2}(t)+2 \operatorname{Li}_{2}(-t)\right. \\
& \left.-\log (t) \log \left(\frac{\left(1+\beta_{\mu}\right)^{3}}{8 \beta_{\mu}^{2}}\right)\right]+3 \log \left(\frac{1-\beta_{\mu}^{2}}{4 \beta_{\mu}}\right)-\log \left(\beta_{\mu}\right) \\
& +\frac{2}{\beta_{\mu}\left(3-\beta_{\mu}^{2}\right)}\left[\frac{3 \beta_{\mu}}{8}\left(5-3 \beta_{\mu}^{2}\right)-\frac{3 \log (t)}{48}\left(33+22 \beta_{\mu}^{2}-7 \beta_{\mu}^{4}\right)\right]
\end{aligned}
$$

is recovered.

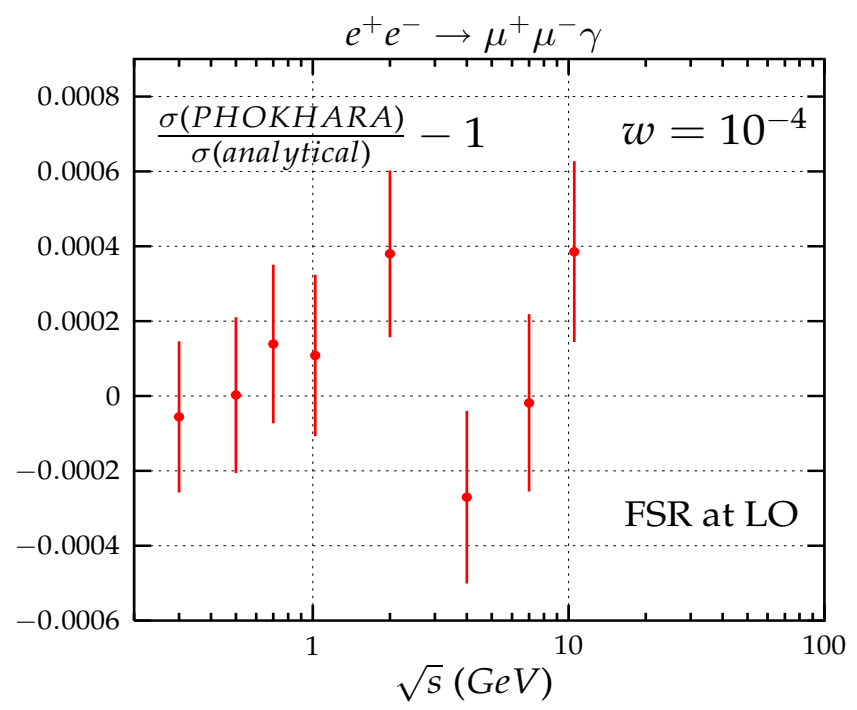

Fig. 3. Comparison between the LO FSR cross section calculated analytically (Eq. (20) and calculated by PHOKHARA for a fixed value of $w=E_{\gamma}^{\min } / \sqrt{s}$. Errors represent one sigma statistical errors.

For single-photon emission from the final state and no further photon radiation, a formula similar to Eq. (16) holds:

$$
\sigma_{\mathrm{FSR}}^{\mathrm{H}}=\frac{\alpha}{\pi} \eta^{\mathrm{H}}\left(s, E_{\gamma}^{\min }\right) \sigma_{e^{+} e^{-} \rightarrow \mu^{+} \mu^{-}}^{(0)}(s),
$$

with $\eta^{\mathrm{H}}$ defined in Eq. (17), $E_{\gamma}^{\mathrm{min}}$ defined now in the $s$ rest frame and $\sigma_{e^{+} e^{-} \rightarrow \mu^{+} \mu^{-}}^{(0)}$ the lowest order cross section of the process $e^{+} e^{-} \rightarrow \mu^{+} \mu^{-}$.

The results of the tests are collected in Figs. 3] and 4 proving the excellent technical precision of this part of PHOKHARA, well below $0.05 \%$. Results of similar tests at NLO are collected in Fig. 5] where results of PHOKHARA are compared with the analytical result of Eq. (19). The latter checks the technical precision of the implementation of double-photon emission, where one photon is emitted from the initial state and the other from the final state, together with the corresponding virtual and soft corrections to the final-state vertex. An agreement of $1 \%$ on the function $\eta$ means in fact an agreement better than $10^{-4}$ on the cross section thanks to the additional factor $\alpha / \pi$ (compare Eq. (9) and Eq. (20)).

The relative size the NLO FSR contributions to the $e^{+} e^{-} \rightarrow \mu^{+} \mu^{-} \gamma(\gamma)$ cross section introduced in PHOKHARA depends on the event selection used. In the following, we will indicate some of its characteristic features. In Fig. 67 the $Q^{2}$ differential cross section is shown with two peaks at low ('soft' muon pair production) and large $Q^{2}$ (soft photon emission) values. In Fig. 6b the corresponding relative NLO FSR contributions are shown. They are $Q^{2}$-dependent and might be as big as a few per cent. As seen by comparison of Figs. 6b and 6r the relative size of this contribution does depend on the event selection used.

At KLOE, the process $e^{+} e^{-} \rightarrow \mu^{+} \mu^{-} \gamma(\gamma)$ is a background to the measurement of the $e^{+} e^{-} \rightarrow \pi^{+} \pi^{-}$cross 

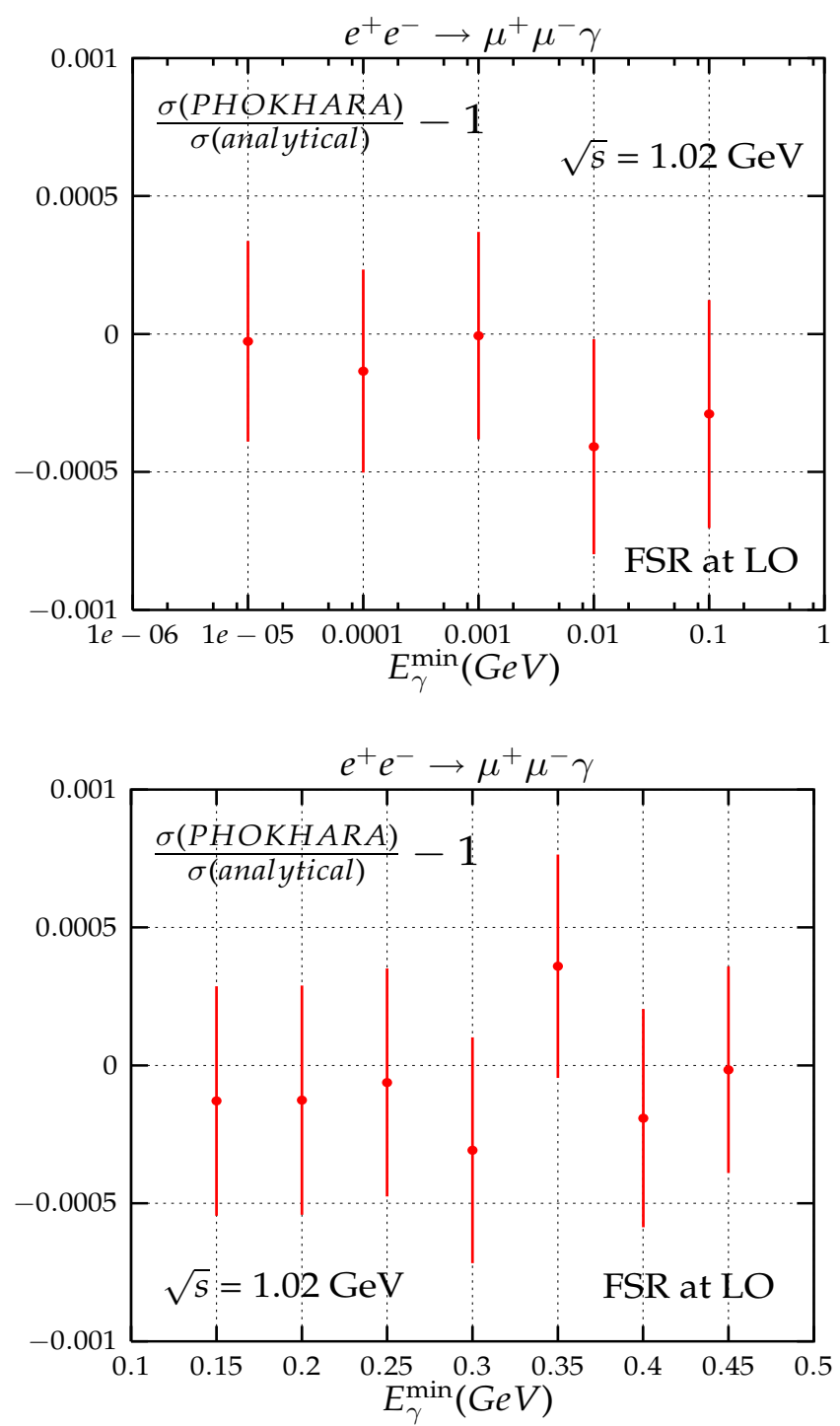

Fig. 4. Comparison between the LO FSR cross section calculated analytically (Eq. (20) and calculated by PHOKHARA for a fixed value of $\sqrt{s}=1.02 \mathrm{GeV}$. Errors represent one sigma statistical errors.

section by the radiative return method, due to possible pion-muon misidentification. The angular cuts used by now by KLOE diminish the low $Q^{2}$ part of the cross section, while the track mass cut, which is specifically introduced to constrain the final state to configurations with $\pi^{+} \pi^{-}$and one photon only (see Ref. 40]), does not allow for events with $\mu^{+} \mu^{-}$and one photon only (see Fig. 17 for corresponding cross sections). If only angular cuts are applied, the newly introduced corrections are at the level of a few per cent (Fig. 7b). However, if in addition the track mass cut is applied, leaving only events with two hard photons, the contribution of one-photon ISR plus onephoton FSR to the two-photon sample is up to $35 \%$ of the two-photon ISR sample (Fig. 7f), thus this contribution is indispensable for a reliable background estimate. The left-over two-hard-photon corrections, with both photons

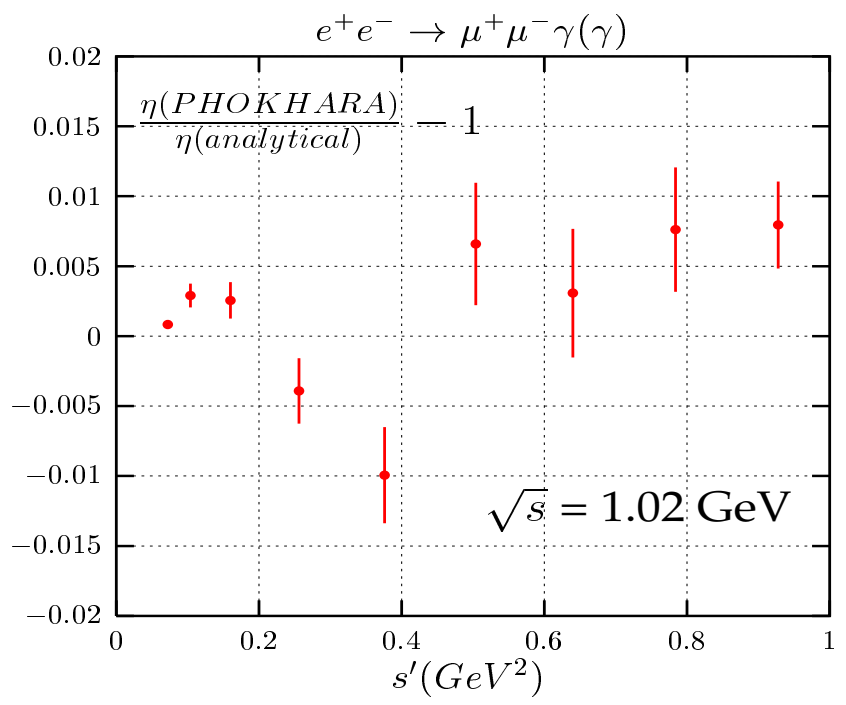

Fig. 5. Comparison between analytical function $\eta$ (Eq. (19)) and $\eta$ obtained from PHOKHARA for $\sqrt{s}=1.02 \mathrm{GeV}$.

emitted from the final state, are expected to be smaller, but will be relevant anyhow if the precision requirement for the background estimate is of the order of a few per cent.

The process $e^{+} e^{-} \rightarrow \mu^{+} \mu^{-} \gamma(\gamma)$ is even more important for BABAR, where it is used as $Q^{2}$-dependent luminosity monitoring. As a result the accuracy requirement is higher. In Fig. 8 some features of the newly implemented corrections are shown in the interesting $Q^{2}$ range, from the point of view of using the radiative return method for measurements of the hadronic cross section. In Fig. [8] the $Q^{2}$ differential cross section is given, including and excluding NLO FSR corrections. As seen from Fig. $8 \mathrm{~b}$ the relative size of those corrections is quite small, of the order of $1 \%$, if no angular cuts are applied; but, as shown in Fig. 81, their relevance depends on the event selection and the corrections can be as big as $10 \%$ for some event selection and $Q^{2}$ range.

\section{ISR corrections to FSR real hard emission for two-pion final state}

Initial-state vertex corrections to FSR for the reaction $e^{+} e^{-} \rightarrow \pi^{+} \pi^{-} \gamma$ (Fig. 9k) were not included in version 3.0 of PHOKHARA, and therefore, for consistency, double photon radiation (Fig. 9b) was restricted to emission of hard ISR. In the present version of the event generator, PHOKHARA 4.0, these corrections [36 have been added and the generation of double photon emission is unconstrained, provided that one of the photons is hard. The virtual and soft corrections to the initial-state vertex are identical to the muon case and Eq. (12) holds, with $d \sigma_{\mathrm{FSR}}^{(0)}$ now being the leading order $e^{+} e^{-} \rightarrow \pi^{+} \pi^{-} \gamma$ cross section, and the photon being emitted off the final pions only.

We report here also formulae analogous to Eqs. (10) and (15), as we have found representations simpler than 

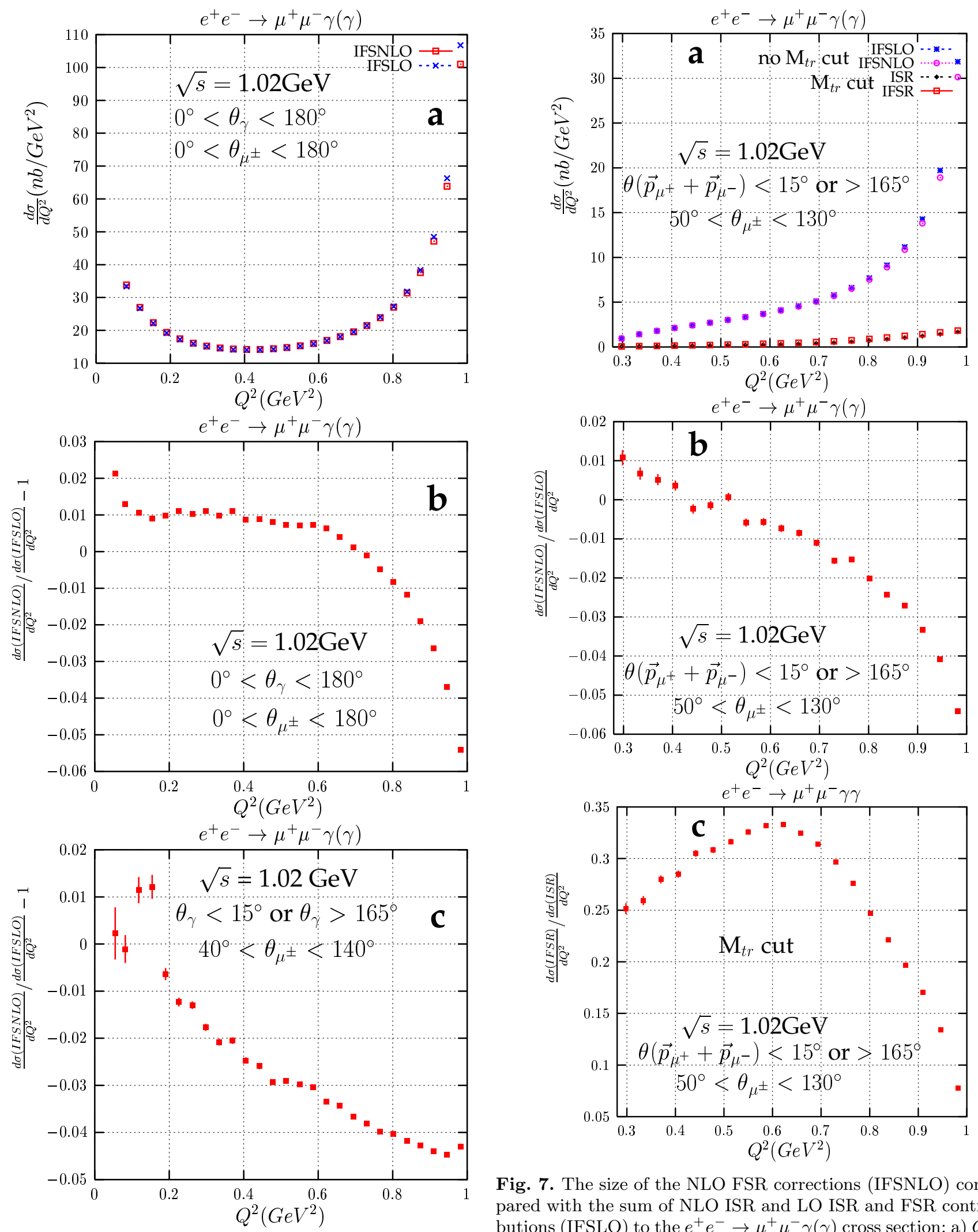

Fig. 7. The size of the NLO FSR corrections (IFSNLO) compared with the sum of NLO ISR and LO ISR and FSR contributions (IFSLO) to the $e^{+} e^{-} \rightarrow \mu^{+} \mu^{-} \gamma(\gamma)$ cross section: a) $Q^{2}$ differential cross sections with angular cuts used by KLOE; b) relative difference of the cross sections with angular cuts used by KLOE (no track mass cut); c) relative difference of the cross sections with angular cuts used by KLOE (with track mass cut). The track mass cut allows only for two-photon events and here IFSR stands for two- and one-photon ISR + one-photon FSR, while ISR stands for two-photon ISR only. 


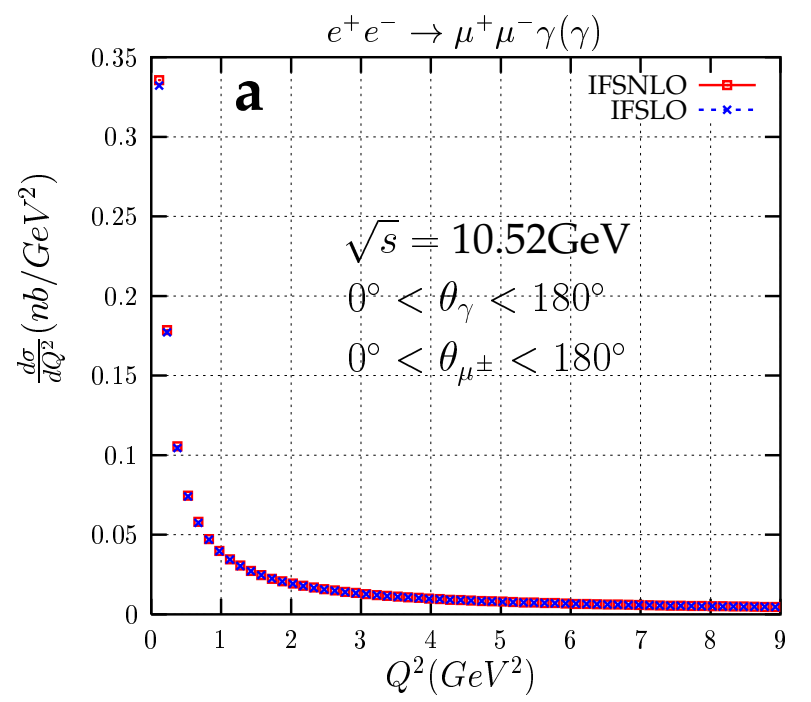

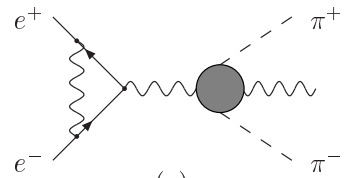

(a)

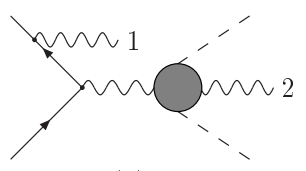

(b)

Fig. 9. Virtual (a) and real (b) radiative corrections to real FSR emission from two-pion final state.

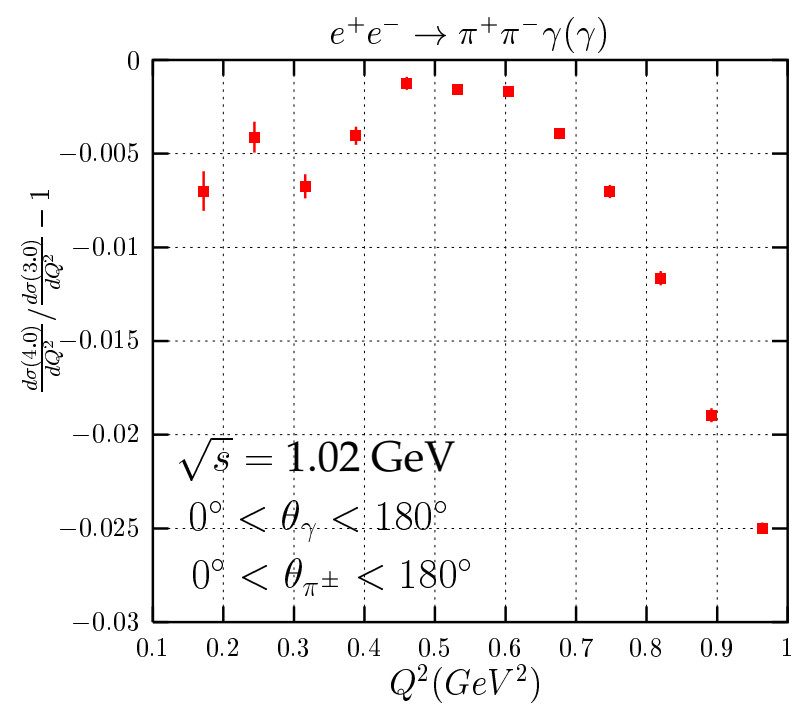

Fig. 10. Comparison between PHOKHARA 3.0 and PHOKHARA 4.0 for $\sqrt{s}=1.02 \mathrm{GeV}$. The pion and photon(s) angles are not restricted.

previously reported in [18. The virtual plus soft corrections, with soft photon energy cut $E_{2}^{\text {cut }}$ in the $s^{\prime}$ rest frame, read

$$
\begin{aligned}
& \eta^{\mathrm{V}+\mathrm{S}}\left(s^{\prime}, E_{2}^{\mathrm{cut}}\right)=-2\left[\frac{1+\beta_{\pi}^{2}}{2 \beta_{\pi}} \log \left(t_{\pi}\right)+1\right] \log (2 w) \\
& -\frac{2+\beta_{\pi}^{2}}{\beta_{\pi}} \log \left(t_{\pi}\right)-2+\log \left(\frac{1-\beta_{\pi}^{2}}{4}\right) \\
& -\frac{1+\beta_{\pi}^{2}}{2 \beta_{\pi}}\left\{-2 \log \left(t_{\pi}\right) \log \left(\frac{1+\beta_{\pi}}{2}\right)\right. \\
& \left.+4 \operatorname{Li}_{2}\left(\frac{2 \beta_{\pi}}{1+\beta_{\pi}}\right)-\pi^{2}\right\}
\end{aligned}
$$

Fig. 8. The size of the next-to-leading FSR corrections (IFSNLO) compared with the sum of NLO ISR and LO FSR contributions (IFSLO) to the $e^{+} e^{-} \rightarrow \mu^{+} \mu^{-} \gamma(\gamma)$ cross section: a) $Q^{2}$ differential cross sections with no angular cuts at $B$ factories; b) relative difference of the cross sections with no angular cuts; c) relative difference of the cross sections with angular cuts resembling the BABAR detector (in $e^{+} e^{-} \mathrm{cms}$ ). If two photons are emitted, both hard (with energy $>100 \mathrm{MeV}$ ) photons are required to be within indicated angular cuts. 


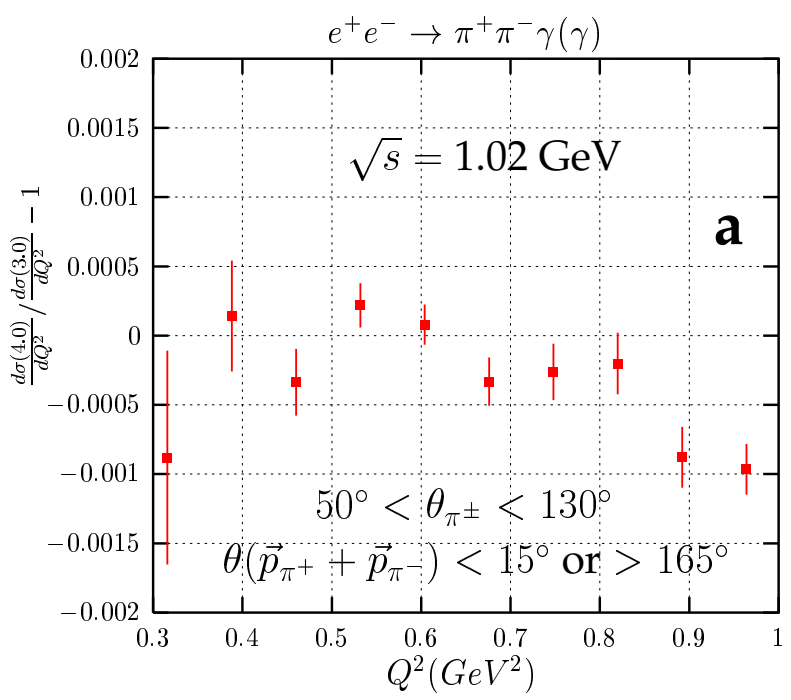

posed they contribute up to $2-3 \%$ to the $Q^{2}$ differential cross section, as shown in Fig. 10 However, for KLOE cuts used for low-angle measurements of the pion form factor, the additional IFSNLO corrections, not included in PHOKHARA 3.0, are well below $0.1 \%$, as anticipated in 18. It is shown in Fig. 11] for event selections with and without the track mass cut. It is worth mentioning that the IFSNLO corrections change also the ratio

$$
\mathcal{R}\left(Q^{2}\right)=\frac{4\left(1+\frac{2 m_{\mu}^{2}}{Q^{2}}\right) \beta_{\mu}}{\beta_{\pi}^{3}\left|F_{\pi}\left(Q^{2}\right)\right|^{2}} \frac{\frac{d \sigma_{\pi}}{d Q^{2}}}{\frac{d \sigma_{\mu}}{d Q^{2}}},
$$

which is expected to be 1 for ISR only, when no cuts are applied. From Figs. 12 and 13] it is clearly visible that one has to take the IFSNLO corrections into account and use a Monte Carlo event generator, when extracting the pion form factor from the ratio of pion and muon cross sections. This is true at both $\Phi$ - and $B$-factories. A similar situation can be expected for other hadronic final states.

\section{Vacuum polarization implementation}

PHOKHARA 4.0 includes also vacuum polarization corrections for all final hadronic states and muon production, with the actual implementation of Ref. 34]. As can be anticipated from Fig. 10 the vacuum polarization corrections are calculated at different scales for different types of diagrams $\left(\hat{Q}^{2}, Q^{2}, s\right.$, etc. $)$. In the energy range that is important for measurements of the components of the hadronic cross section through the radiative return method, the vacuum polarization corrections have a non-trivial behaviour (see Fig. 14) due to the hadronic contributions. For event selections, for which only ISR corrections are important, the vacuum polarization contribution is just a multiplicative factor depending on $Q^{2}$, so that one can correct the event generator results even after generation. However, for event selections where other contributions are also important, event generation with complete implementation of vacuum polarization might become necessary. An example is shown in Fig. 15] where the vacuum polarization contributions do change the forward-backward asymmetry of the pion polar angle distribution. The effect, of up to $5 \%$, is mainly caused by the choice of $s$ in the close vicinity of the $\Phi$ resonance, which is nothing but the KLOE case. As seen in Fig. 14 the vacuum polarization around the $\Phi$ is much larger than at lower energies. As a result, the vacuum polarization correction to the ISR-FSR interference, which contributes to the numerator of the asymmetry, is larger than the corresponding corrections to its denominator, which is dominated by ISR. Therefore, vacuum polarization corrections do not drop in the ratio. This effect has to be taken into account, when studies of model dependence of FSR are performed.

where $w=E_{\gamma}^{\min } / \sqrt{s}$.

\section{Summary and Conclusions}

The numerical importance of the corrections from Fig. 9 Precision measurements of the hadronic cross section through depends on the event selection. If no angular cuts are imthe radiative return to an accuracy of about $1 \%$ require the 

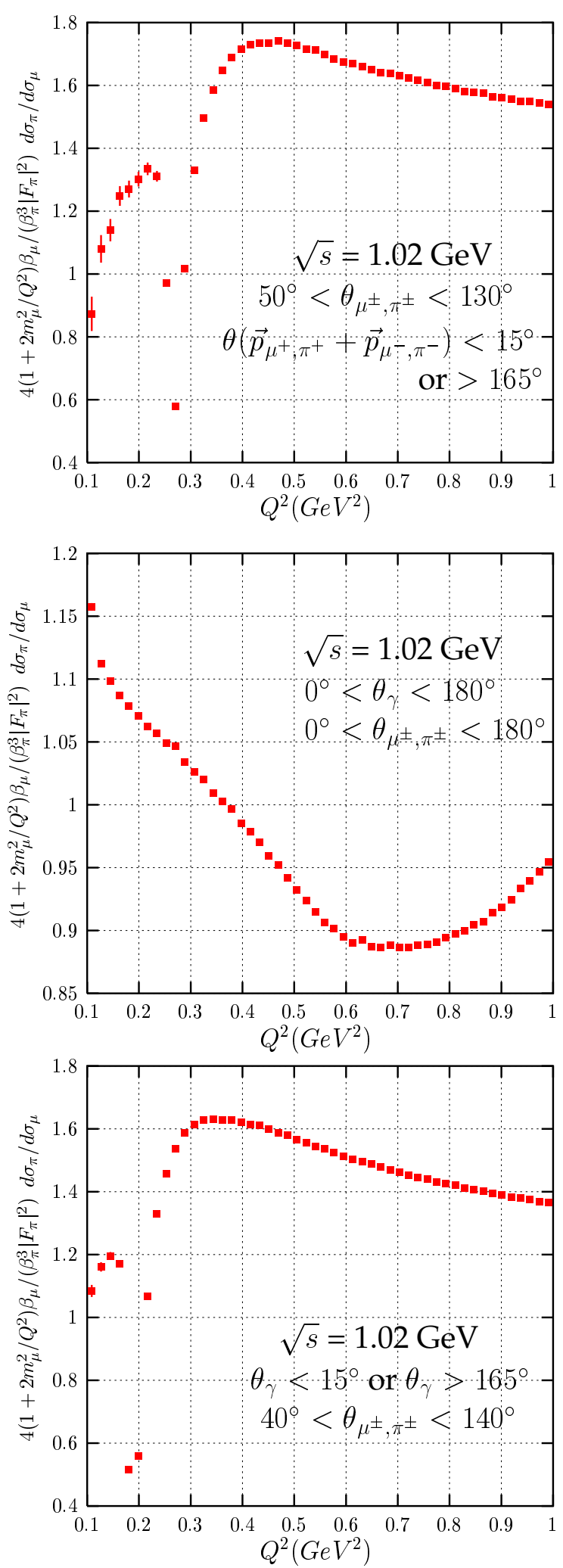

Fig. 12. The ratio $\mathcal{R}$ of Eq. (23) for $\sqrt{s}=1.02 \mathrm{GeV}$, for three different event selections.
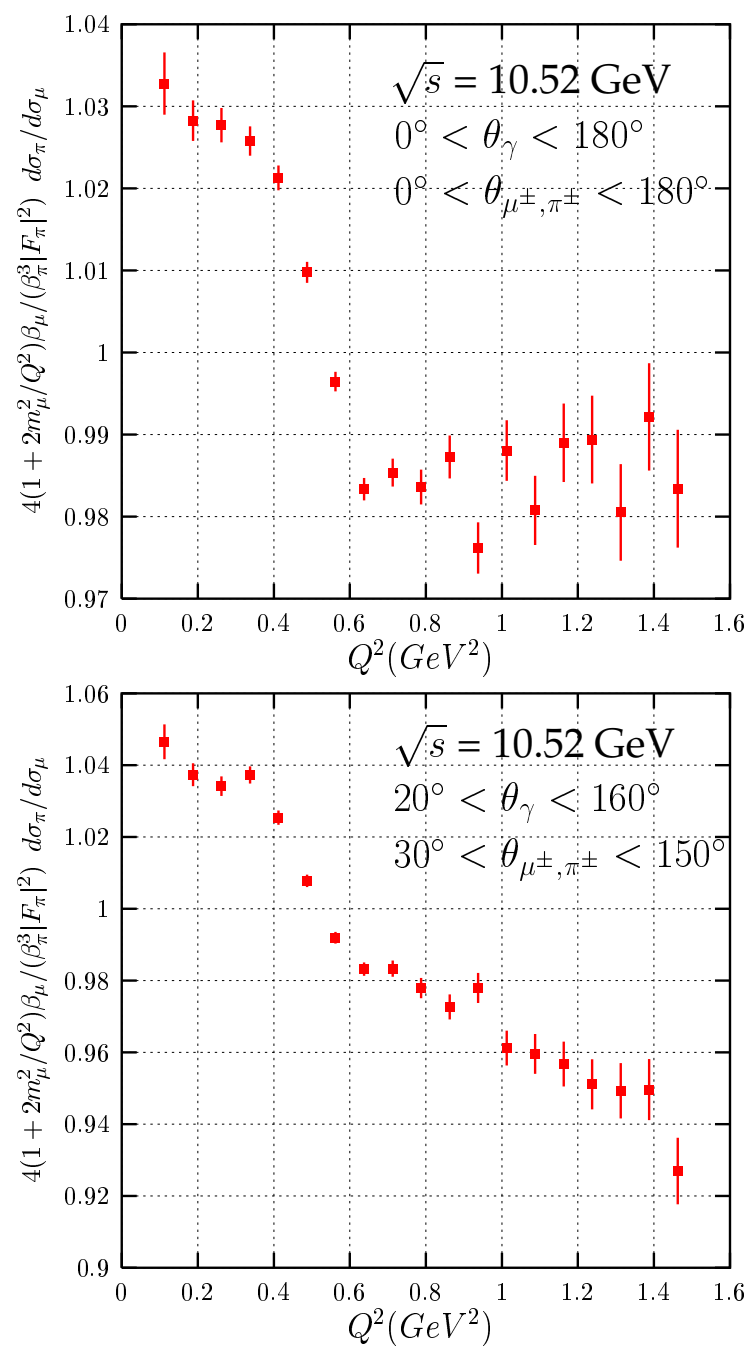

Fig. 13. The ratio $\mathcal{R}$ of Eq. (23) for $\sqrt{s}=10.52 \mathrm{GeV}$ and two different event selections.

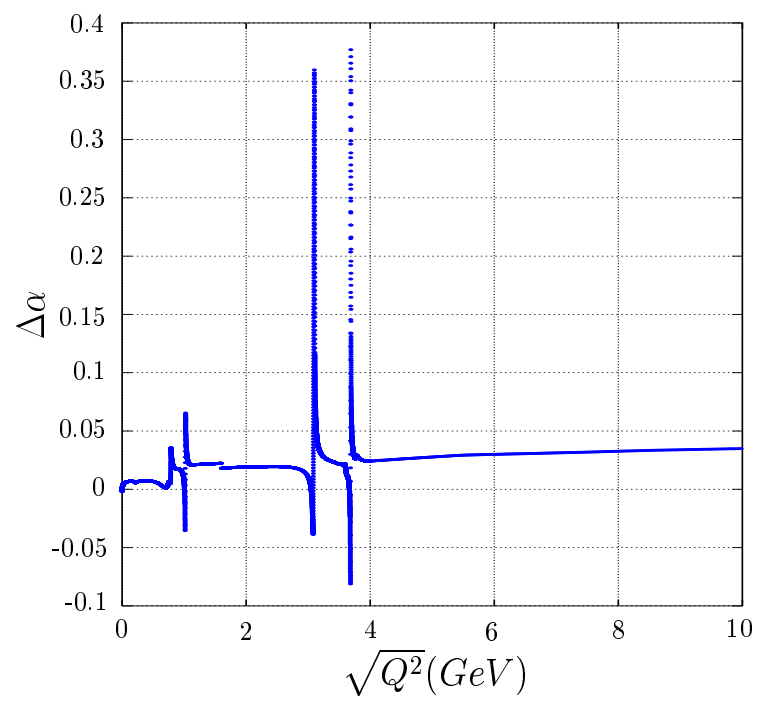

Fig. 14. The energy dependence of $\Delta \alpha$ calculated by the program of Ref. 34. 


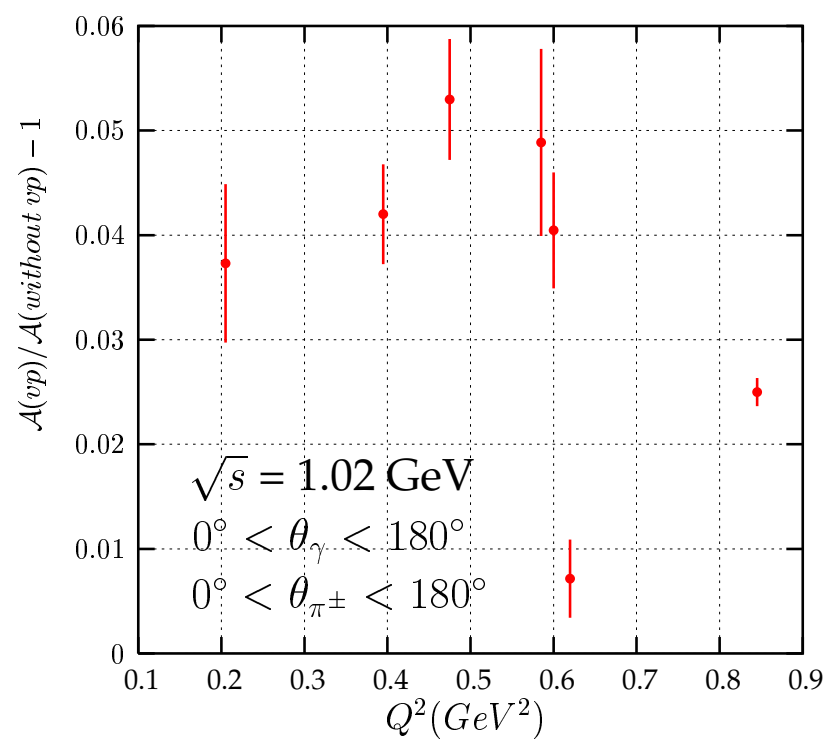

Fig. 15. The relative difference of the forward-backward asymmetry of the pion polar angle distribution, calculated with and without vacuum polarization contributions.

full understanding of radiative corrections and their implementation in a Monte Carlo event generator. For this reason a number of new features have been introduced into the Monte Carlo event generator PHOKHARA.

In a first step we presented the formulae for real and virtual radiation, which are required to describe FSR for muon pair production through the radiative return in nextto-leading order. This includes a specific set of virtual corrections as well as real radiation. The Monte Carlo event generator PHOKHARA has been upgraded and, in its present form, also simulates the contribution from the "two-step" reaction $e^{+} e^{-} \rightarrow \gamma \gamma^{*}\left(\rightarrow \mu^{+} \mu^{-}\right)$. The technical precision of the generator in this new mode has been demonstrated to be better than $10^{-4}$. It is shown that the relative importance of this NLO correction depends strongly on the details of the experimental cuts.

The program has also been extended to the case of pion production. In particular corrections from virtual plus soft ISR, in combination with hard FSR, have been introduced. Although the impact of this additional piece is small, as far as actual measurements at KLOE or the $B$ factories are concerned, it is required for a complete NLO treatment of the radiative return for pion-pair production. Another straightforward upgrade is the introduction of vacuum polarization in the photon propagator. As far as charge-symmetric cross section measurements are concerned, this effect can just be considered as a multiplicative, $Q^{2}$-dependent change of the normalization. However, interference terms between amplitudes with virtual photons of different virtualities, which are relevant to e.g. the forward-backward asymmetry, do lead to modifications of the results at the level of several per cent.

\section{Acknowledgements}

We would like to thank A. Denig, W. Kluge, D. Leone and S. Müller for discussions of the experimental aspects of our analysis. H.C. and A.G. are grateful for the support and the kind hospitality of the Institut für Theoretische Teilchenphysik of the Universität Karlsruhe. Special thanks to Suzy Vascotto for careful proof-reading of the manuscript.

\section{References}

1. Min-Shih Chen and P. M. Zerwas, Phys. Rev. D 11 (1975) 58 .

2. S. Binner, J. H. Kühn and K. Melnikov, Phys. Lett. B 459 (1999) 279 hep-ph/9902399.

3. H. Czyż and J. H. Kühn, Eur. Phys. J. C 18 (2001) 497 hep-ph/0008262.

4. H. Czyż, A. Grzelińska, J. H. Kühn and G. Rodrigo, Eur. Phys. J. C 27 (2003) 563 hep-ph/0212225.

5. H. Czyż, J. H. Kühn, E. Nowak and G. Rodrigo, Eur. Phys. J. C 35, 527 (2004) hep-ph/0403062.

6. G. Rodrigo, H. Czyż, J.H. Kühn and M. Szopa, Eur. Phys. J. C 24 (2002) 71 hep-ph/0112184.

7. H. Czyż and E. Nowak, Acta Phys. Polon. B 34 (2003) 5231 hep-ph/0310335.

8. G. Rodrigo, A. Gehrmann-De Ridder, M. Guilleaume and J. H. Kühn, Eur. Phys. J. C 22 (2001) 81 hep-ph/0106132.

9. J. H. Kühn and G. Rodrigo, Eur. Phys. J. C 25 (2002) 215 hep-ph/0204283.

10. A. Aloisio et al. [KLOE Collaboration], hep-ex/0307051

11. S. Di Falco [KLOE Collaboration], Acta Phys. Polon. B 34 (2003) 5207 hep-ex/0311006.

12. A. G. Denig [KLOE Collaboration], hep-ex/0311012

13. A. Aloisio et al. [KLOE Collaboration], eConf C0309101, FRWP008 (2003) hep-ex/0312056.

14. M. Davier, hep-ex/0312063

15. G. Rodrigo, Acta Phys. Polon. B 32 (2001) 3833 hep-ph/0111151.

16. J. H. Kühn, Nucl. Phys. Proc. Suppl. 98 (2001) 289 hep-ph/0101100.

17. G. Rodrigo, H. Czyż and J. H. Kühn, hep-ph/0205097 Nucl. Phys. Proc. Suppl. 123 (2003) 167 hep-ph/0210287; Nucl. Phys. Proc. Suppl. 116 (2003) 249 hep-ph/0211186.

18. H. Czyż, A. Grzelińska, J. H. Kühn and G. Rodrigo, Eur. Phys. J. C 33 (2004) 333 hep-ph/0308312.

19. H. Czyż and A. Grzelińska, Acta Phys. Polon. B 34 (2003) 5219 hep-ph/0310341.

20. H. Czyż and A. Grzelińska, eConf C0309101 (2003) FRWP007 hep-ph/0402030.

21. G. Cataldi, A. Denig, W. Kluge, S. Muller and G. Venanzoni, Frascati Physics Series (2000) 569.

22. M. Adinolfi et al. [KLOE Collaboration], hep-ex/0006036

23. A. Aloisio et al. [KLOE Collaboration], hep-ex/0107023

24. A. Denig et al. [KLOE Collaboration], eConf C010430 (2001) T07 hep-ex/0106100.

25. B. Valeriani et al. [KLOE Collaboration], hep-ex/0205046

26. N. Berger, eConf C020620 (2002) THAP10 hep-ex/0209062. 
27. G. Venanzoni et al. [KLOE Collaboration], eConf C0209101 (2002) WE07 hep-ex/0210013; hep-ex/0211005

28. A. Blinov, talk at International Conference 'New Trends in High-energy Physics' Alushta, Crimea (May 2003) http://www.slac.stanford.edu/ ablinov/

29. J. Lee-Franzini, talk at Lepton Int. Sym. 2003, http://g2pc1.bu.edu/leptonmom/

30. A. G. Denig et al. [KLOE Collaboration], eConf C0309101 (2003) FRWP009 hep-ex/0403005.

31. A. Hoefer, J. Gluza and F. Jegerlehner, Eur. Phys. J. C 24 (2002) 51 hep-ph/0107154.

32. J. Gluza, A. Hoefer, S. Jadach and F. Jegerlehner, Eur. Phys. J. C 28 (2003) 261 hep-ph/0212386.

33. H. Czyż, A. Grzelińska, J. H. Kühn and G. Rodrigo, Nucl. Phys. Proc. Suppl. 131 (2004) 39 hep-ph/0312217.

34. F. Jegerlehner, http://www-zeuthen.desy.de/ fjeger/alphaQED.uu

35. F. A. Berends, K. J. F. Gaemer and R. Gastmans, Nucl. Phys. B 57 (1973) 381

36. F. A. Berends, W. L. van Neerven and G. J. H. Burgers, Nucl. Phys. B 297 (1988) 429 [Erratum-ibid. B 304 (1988) 921].

37. S. Jadach, B.F.L. Ward and Z. Wạs, Comput. Phys. Commun. 130 (2000) 260 hep-ph/9912214.

38. J. A. M. Vermaseren, Symbolic Manipulations with FORM, Computer Algebra Nederland, Amsterdam, 1991.

39. K. G. Chetyrkin, J. H. Kühn and A. Kwiatkowski, Phys. Rep. 277 (1996) 189.

40. A. G. Denig et al. [the KLOE Collaboration], Nucl. Phys. Proc. Suppl. 116 (2003) 243 hep-ex/0211024. 